\title{
Acute ST-elevation myocardial infarction due to prosthetic valve endocarditis after transcatheter aortic valve implantation
}

\author{
Jun Hwan Cho, Jung-Kyu Han, Han-Mo Yang, Bon-Kwon Koo, and Hyo-Soo Kim
}

Department of Internal Medicine and Cardiovascular Center, Seoul National University Hospital, Seoul, Korea

Received: October 4, 2018

Revised : December 10, 2018

Accepted: January 20, 2019

\section{Correspondence to}

Hyo-Soo Kim, M.D.

Tel: +82-2-2072-2226

Fax: $+82-2-766-8904$

E-mail: hyosoo@snu.ac.kr
An 8o-year-old man was admitted to our hospital with a 3-week history of fever. The patient was treated with transcatheter aortic valve implantation (TAVI; $23 \mathrm{~mm}$ LOTUS, Boston Scientific Corp., Natick, MA, USA) 23 months before the current admission. He had a history of chronic obstructive pulmonary disease, myocardial infarction, and chronic kidney disease. Five weeks prior to admission, he had been diagnosed with scrub typhus, which improved after a week of doxycycline treatment.

Blood cultures were positive for methicillin-resistant Staphylococcus epi-
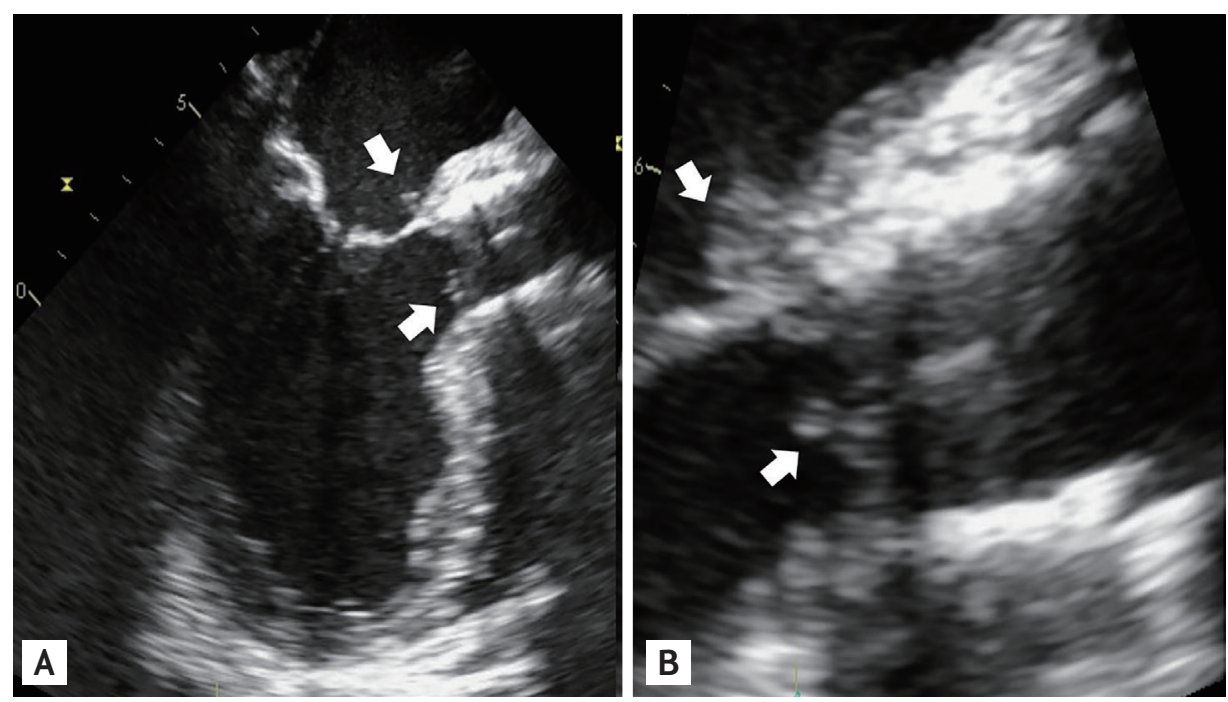

Figure 1. (A, B) These shows the non-mobile vegetations (arrows) measuring $0.9 \times 0.4 \mathrm{~cm}$ on the transcatheter aortic valve implantation prosthesis and atrial side of mitral valve in transesophageal echocardiogram. 

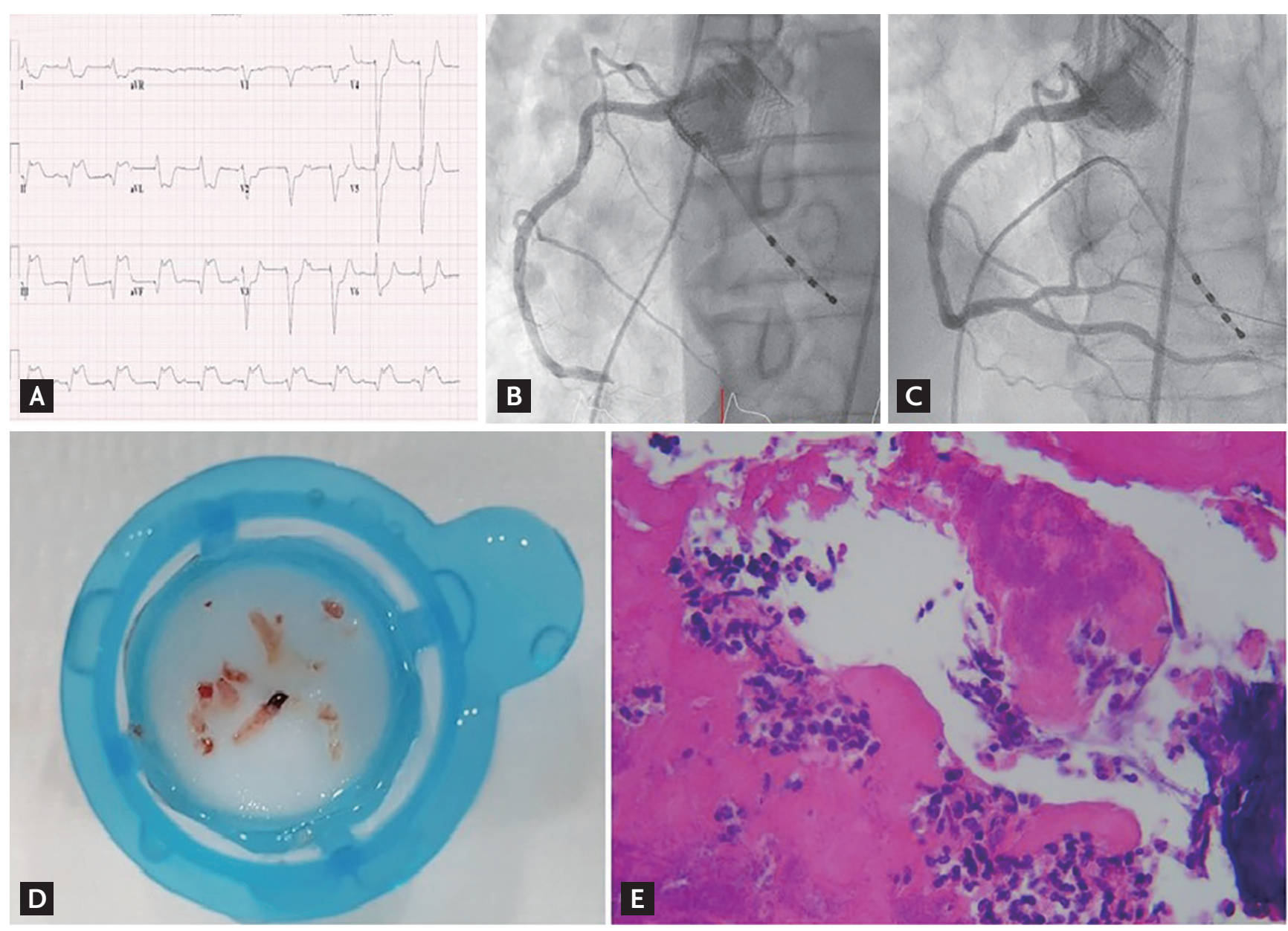

Figure 2. (A) It shows the 12-lead electrocardiogram with ST-elevation in leads II, III, and aVF. (B) It shows complete occlusion of the distal right coronary artery. (C) It shows recovered right coronary artery flow after thrombectomy. (D) It shows multiple thrombi-vegetations. (E) It shows an embolic vegetation retrieved from the right coronary artery. Bacterial clumps and neutrophil infiltration are noted on histopathological examination $(\mathrm{H} \& \mathrm{E}, \times 400)$.

was restored (Fig. 2C, Supplementary Video 3) and many thrombi were retrieved (Fig. 2D). Moreover, in the thrombus culture, MRSE was identified. Bacterial clumps and neutrophil infiltration were seen on histological examination (Fig. 2E).

He was continuously treated with antibiotics and dual antiplatelet agents. Surgery was declined due to the patient's poor condition, multiple comorbidities, and persistent bacteremia. Over the next few days, the patient developed multiple systemic emboli causing renal and bowel infarctions. Despite meticulous parenteral antibiotic treatment, the patient died due to multiple organ failure.

With the increase in the number of TAVI cases worldwide, the frequency of post-TAVI endocarditis will in- crease. Post-TAVI endocarditis can manifest in various forms. Embolic vegetations associated with ST elevation are rare, and a high degree of clinical suspicion and accurate diagnosis are important for the treatment. Given the serious baseline condition of patients treated using TAVI and the high fatality of infective endocarditis, careful management is warranted before and after the procedure.

The patient provided written informed consent for participation.

\section{Conflict of interest}

No potential conflict of interest relevant to this article was reported. 
KJIM

The Korean Journal of Internal Medicine. Vol. 35, No. 4, July 2020

Supplementary Video 1. $0.9 \mathrm{X} 0.4 \mathrm{~cm}$ non-mobile vegetations both on the TAVI prosthesis and the atrial side of mitral valve.

Supplementary Video 2. Complete occlusion of the distal right coronary artery in coronary angiogram.

Supplementary Video 3. After repeated thrombosuction, the coronary flow was restored. 